\title{
Evaluating the Clinical Application Value of Ferric Citrate Effervescent Granules (FAC) in Magnetic Resonance Cholangio-Pancreatography (MRCP) Inspection
}

\author{
Sihua Liang, Xiangran Cai, Nengwei Wang* \\ Radiology Department, The First Affiliated Hospital, Jinan University, Guangzhou, China \\ Email address: \\ 13533123111@139.com (Sihua Liang), caixran@163.com (Xiangran Cai), 1436295666@qq.com (Nengwei Wang) \\ ${ }^{*}$ Corresponding author
}

To cite this article:

Sihua Liang, Xiangran Cai, Nengwei Wang. Evaluating the Clinical Application Value of Ferric Citrate Effervescent Granules (FAC) in Magnetic Resonance Cholangio-Pancreatography (MRCP) Inspection. International Journal of Medical Imaging.

Vol. 8, No. 3, 2020, pp. 45-48. doi: 10.11648/j.ijmi.20200803.12

Received: August 3, 2020; Accepted: August 19, 2020; Published: August 27, 2020

\begin{abstract}
Objective: Assessing the clinical application value of Ferric Citrate Effervescent granules (FAC) in magnetic resonance cholangio-pancreatography (MRCP) inspection. Methods: We invited 10 patients who were diagnosed with diseases of the pancreas system and bile duct system and undergoing magnetic resonance cholangio-pancreatography and use Ferric Citrate Effervescent granules in the MRCP process. The time of their MRCP is from May 2018 to February 2020. In addition, we collected the information from the participants, that the information included Sequence and scanning parameters of $\mathrm{Mr}$ Cholangiopancreatography, Image quality score before and after MRCP administration, contrast to noise ratio (CNR) score and comparison score of different parts in MRCP images before and after drug administration. Result: In scanning parameters, the data is standard in research, that the TR and TE of 2d MRCP and 3D MRCP are similar, that are $>6000$ and $>500$. But Layer thickness/Layer spacing between the objects had big gap in data, that are 50/0 and 1.8/0. Additionally, FOV had little different length, which is 40 vs 36 . In NEX, they are same. In image quality score, it has higher score after taking the FAC. The CNR score research is similar, that FAC affect the CNR outcome. Conclusion: the FAC provide the improvement to the outcome of patient in MRCP process, that it improves image quality of MRCP.
\end{abstract}

Keywords: Magnetic Resonance Cholangio-Pancreatography, Ferric Citrate Effervescent Granules, Image Quality

\section{Introduction}

In medical imaging, the magnetic resonance cholangio-pancreatography (MRCP) is common detection method. Base on the research, MRCP is a noninvasive imaging technique, that it is often used to establish role in demonstrating biliary tract anatomy and in providing a roadmap for interventional treatments [1-3]. As the development of science and technology in medical electronics, the quality of the MRCP images was improved by many three-dimensional (3D) sequences. In 3D MRCP, it uses a maximum intensity projection or volume rendered technique for accurate display of the biliary tree and pancreatic duct [4-6]. In limitation, as the adoption of heavy T2 weighted MRCP imaging, images of gastrointestinal fluid often overlap with the pancreatic bile duct when doctor use MRCP to test the patient. As a result, the bile ducts of the pancreas are not clearly visible [7]. However, during MRCP examination, simultaneous development of gastric and intestinal fluids showed high signals, which interfered with the display of biliary and pancreatic ducts. In Chinese hospital, the doctor often uses ferric ammonium citrate granules (FAC) to improve image quality of MRCP $[15,17]$.

Ferric ammonium citrate granules are commonly used as blood tonic in clinic to treat iron deficiency anemia [8]. It is mainly composed of iron, ammonium and citric acid, and varies with different synthetic conditions, so there is no accurate chemical formula. Meanwhile, ammonium ferric citrate has long been studied and used as a water-soluble positive contrast agent [9]. The active component which contains 5 unpaired spintronic paramagnetic iron elements, and its characteristics can reduce the T2 value of gastric and 
duodenal fluid shortening, thus reducing the interference background caused by its high signal [10]. The aim of this study is evaluating the clinical application value of Ferric Citrate Effervescent granules (FAC) in magnetic resonance cholangio-pancreatography (MRCP) inspection.

\section{Methods}

\subsection{Participants Enrollment and Survey Methods}

We invited 10 patients who were diagnosed with diseases of the pancreas system and bile duct system and undergoing magnetic resonance cholangio-pancreatography and use Ferric Citrate Effervescent granules in the MRCP process. The time of their MRCP is from May 2018 to February 2020. All participants had been signed informed consent before this research beginning. In addition, we collected the information from the participants, that the information included Sequence and scanning parameters of Mr Cholangiopancreatography, Image quality score before and after MRCP administration, CNR score and comparison score of different parts in MRCP images before and after drug administration.

By two with one high qualification doctor before and after oral FAC image quality assessment rating: MRCP I level: a large patch of high signal within the gastrointestinal tract shadow liquid artifact is serious, affect the diagnosis (1); II part of the gastrointestinal fluid artifact implications to the diagnosis ( 2 points); III level: there are a few gastrointestinal fluid artifact, but will not affect the diagnosis ( 3 points); IV, completely inhibited gastrointestinal fluid signal (4 points). Add the scores of the two reviewers and divide by 2 as the final score

Their inclusion criteria were: (1) The patient successfully complete the MRCP (2) Patients volunteered to participate in research. (3) They did not suffer from other severe complications. Their withdraw criteria were: (1) Poor mental health (2) Changing hospital during investigation.

\subsection{Statistical Analysis}

Our data analyzer performed the statistical analysis by SPSS 22.0. The $\mathrm{P}$ value, t-test and chi-square test were associated with collection result were analyzed.

\section{Result}

In sequence and scanning parameters of $\mathrm{Mr}$ cholangiopancreatography, the data include TR (ms), TE (ms), layer thickness/Layer spacing $(\mathrm{mm})$, FOV (cm) and NEX (Table 1). The TR and TE of 2d MRCP and 3D MRCP are similar, that are $>6000$ and $>500$. But Layer thickness/Layer spacing between the objects had big gap in data, that are 50/0 and 1.8/0. Additionally, FOV had little different length, which is 40 vs 36 . In NEX, they are same.

Table 1. Sequence and scanning parameters of Mr cholangiopancreatography.

\begin{tabular}{|c|c|c|c|c|c|}
\hline Projects & TR (ms) & TE (ms) & Layer thickness/Layer spacing (mm) & FOV (cm) & NEX \\
\hline 2D MRCP & $>6000$ & $>500$ & $50 / 0$ & 40 & 1 \\
\hline 3D MRCP & $>6000$ & $>500$ & $1.8 / 0$ & 36 & 1 \\
\hline
\end{tabular}

In image quality score before and after MRCP administration, it contains two parts that are 2D MRCP and 3DMRCP. In the part it has the before taking the medicine step and the after taking the medicine step. In between two steps, the after taking the medicine step has higher score. In between two parts, II level has higher score in research (Table 2).

Table 2. Image quality score before and after MRCP administration.

\begin{tabular}{|c|c|c|c|c|c|c|c|c|c|c|}
\hline \multirow{2}{*}{ Projects } & \multicolumn{5}{|c|}{ 2D MRCP } & \multicolumn{5}{|c|}{ 3D MRCP } \\
\hline & I level & II level & III level & IV level & score & I level & II level & III level & IV level & score \\
\hline Before taking the medicine & 2 & 7 & 1 & 0 & 19 & & 4 & 5 & 1 & 0 \\
\hline After taking the medicine & 0 & 0 & 3 & 7 & 37 & & 0 & 0 & 2 & 8 \\
\hline $\mathrm{Z}$ & -3.849 & & & & & -3.880 & & & & \\
\hline
\end{tabular}

In Table 3, it indicated contrast to noise ratio (CNR) score of different parts in before and after taking the medicine. In after taking the medicine research, it has better performance than that of before taking the medicine (FAC). In the result, the bottom of stomach has biggest changing between before using the FAC and after using the FAC (from 211.39 \pm 103.60 to $81.75 \pm 98.07$ ). Additionally, only duodenum and right hepatic duct are statistical significance in the research result $(\mathrm{P}<0.05)$

Table 3. CNR score and comparison of different parts in MRCP images before and after drug administration.

\begin{tabular}{llllll}
\hline Projects & & Before & After & t & P \\
\hline \multirow{3}{*}{ stomach } & Bottom & $211.39 \pm 103.60$ & $81.75 \pm 98.07$ & 5.54 & 0.00 \\
& Body & $58.00 \pm 79.53$ & $14.69 \pm 39.99$ & 1.68 & 0.13 \\
The gall bladder & Sinus & $21.01 \pm 32.70$ & $12.87 \pm 32.02$ & 0.75 & -0.72 \\
& & $182.20 \pm 100.10$ & $217.81 \pm 204.90$ & 2.70 & 0.48 \\
duodenum & Spheric part & $57.74 \pm 52.20$ & $17.41 \pm 28.11$ & 2.70 & 1.89 \\
& Descending part & $97.46 \pm 65.64$ & $36.82 \pm 40.60$ & 0.49 & 0.02 \\
jejunum & Standard part & $21.41 \pm 35.51$ & $4.31 \pm 11.89$ & 0.02 & 0.09 \\
\hline
\end{tabular}




\begin{tabular}{llllll}
\hline Projects & & Before & After & t & P \\
\hline & left hepatic duct & $57.31 \pm 28.19$ & $69.28 \pm 63.46$ & -0.84 & 0.42 \\
Bile duct & right hepatic duct & $78.36 \pm 62.03$ & $48.88 \pm 35.50$ & 2.22 & 0.05 \\
& Hepatic duct & $61.35 \pm 33.75$ & $53.99 \pm 27.87$ & 0.64 & 0.54 \\
pancreatic duct & Common bile duct & $79.90 \pm 51.91$ & $95.70 \pm 71.72$ & -0.80 & 0.45 \\
\hline
\end{tabular}

Before $=$ before using the FAC

After $=$ after using the FAC

\section{Discussion}

The medical imaging, the magnetic resonance cholangio-pancreatography is defined that is a non-invasive, safe and effective cholangiopancreatography technique that uses the heavy T2-weighted technique to present high signals in the anatomical structure of the pancreatic bile duct containing bile and pancreatic fluid, and no contrast agent is used in the imaging process [11-13]. It is a non-invasive, safe and effective cholangiopancreatography technique [14]. In 2D-MRCP, its thick collection sequence through single level, without post-processing gain levels within the system volume of pancreatic MRCP projection images, often in a breath breathless cases at the end of the collection. Although the clinical commonly used 2D-MRCP, 3D-MRCP sequence is also a kind of method cannot be ignored, it is a way of $3 \mathrm{~d}$ acquisition, collect data by thin layer scanning, and the post-processing can be directly from different angles show the anatomical structure of the system of pancreatic $[15,16]$.

According to the results above, the FAC provide the improvement to the outcome of patient in MRCP process, that it improves image quality of MRCP. In Table 2 , it indicated FAC increase the image quality score in $2 \mathrm{D}$ MRCP group and $3 \mathrm{D}$ MRCP group, that FAC improve the image quality so that the MRCP effect is improved. In addition, CNR scores are decreased after FAC administration, it indicated FAC can affect the CNR in different body parts. But only duodenum and right hepatic duct are statistical significance in the research result $(\mathrm{P}$ $<0.05)$. In limitation, CNR was decreased in all parts, but only in the stomach and duodenum was statistically significant in this paper, which may be related to the small number of samples, which should be further examined after the sample size was enlarged. In addition, the sample size also is a limitation in this study, that most CNR score are not statistical significance in the result as samples are too less.

\section{Conclusion}

In conclusion, the FAC provide the improvement to the outcome of patient in MRCP process, that it improves image quality of MRCP. In study result, image quality score had higher score after taking the FAC. In addition, the FAC only action on the he bottom of stomach, duodenum and right hepatic duct as their CNR score are statistical significance. However, CNR was decreased in all parts, but only in the stomach and duodenum was statistically significant in this paper, which may be related to the small number of samples, which should be further examined after the sample size was enlarged.

\section{References}

[1] Mungai F, Berti V, Colagrande S. Bile leak after elective laparoscopic cholecystectomy: role of MR imaging. J Radiol Case Rep. 2016; 7: 25-32.

[2] Schofer JM. Biliary causes of post cholecystectomy syndrome. J Emerg Med. 2015; 39: 406-10.

[3] Terhaar OA, Abbas S, Thornton FJ, Duke D, O'Kelly P, Abdullah $\mathrm{K}$. Imaging patients with post-cholecystectomy syndrome: an algorithmic approach. Clin Radiol. 2015; 60: 78-84.

[4] Gulati K, Catalano OA, Sahani DV. Review: advances inmagnetic resonance cholangiopancreatography: from morphology to functional imaging. Indian J Radiol Imaging. 2017; 17: 247-255

[5] Rastegar N, Matteoni-Athayde LG, Eng J, Takahashi N, Tamm $\mathrm{EP}$, Mortele KJ, et al. Incremental value of secretin-enhanced magnetic resonance cholangiopancreatography in detecting ductal communication in a population with high prevalence of small pan-creatic cysts. Eur J Radiol 2015; 84 (4): 575-580.

[6] Boninsegna E, Manfredi R, Ventriglia A, Negrelli R, Pedrinolla $\mathrm{B}$, Mehrabi S, et al. Santorinicele: secretin-enhanced magnetic resonance cholangio-pancreatography findings before and after minor papilla sphincterotomy. Eur Radiol. 2015; 25 (8): 2437-2444.

[7] Kim JH, Hong SS, Eun HW, Han JK, Choi BI. Clinical usefulness of free-breathing navigator-triggered 3D MRCP in non-cooperative patients: comparison with con-ventional breath-hold 2D MRCP. Eur J Radiol. 2012; 81 (4): 513-518.

[8] Taourel P, Bret P M, Reinhold C, et al. Anatomic variants of the biliary tree: diagnosis with MR cholangiopancreatography. Radiology. 1996.

[9] Broglia L, Tortora A, Maccioni F, Arpesani R, Marcelli G, Ascarelli A, Rossi P. Optimization of dosage and exam technique in the use of oral contrast media in magnetic resonance. La Radiologia medica. 1999.

[10] Xu XM, liang F, Gao GM. Clinical value analysis of oral ferric ammonium citrate effervescent particles in MRCP in gallbladder duct variation. Forum of primary medicine. 2016, 20 (32): 4577-4579.

[11] Liu N, Huang XF, Dong GL, et al. Progress of MRCP on clinical research of pancreatic duct system and variations. Journal of North Sichuan Medical College, 2015, 30 (3): 418-422.

[12] Pan H. Application value of 3D-SPACE and 3D-TSE sequence MRCP in the diagnosis of gallbladder and choledocholithiasis [J]. Journal of Imaging Research and Medical Applications. 2019, 3 (15): 30-32. 
[13] Yoon JH, Lee SM, Kang HJ, et al. Clinical feasibility of 3-dimensional magnetic resonance cholangiopancreatography using compressed sensing: comparison of image quality and diagnostic performance. Investigative Radiology, 2017, 52 (10): 612-619.

[14] Tokoro H, Yamada A, Suzuki T, et al. Usefulness of breath-hold compressed sensing accelerated three-dimensional magnetic resonance cholangiopancreatography (MRCP) added to respiratory-gating conventional MRCP. European Journal of Radiology. 2020; 122: 108765.
[15] Zhu L, Sun ZY, Xue HD, et al. Patient-adapted respiratory training: Effect on navigator-triggered 3D MRCP in painful pancreatobiliary disorders. Magnetic Resonance Imaging. 2018; 45: 43-50.

[16] Kong YL, QIang YQ, Dong BM, et al. Diagnostic efficacy of 3. 0T MR 2D and 3D MRCP sequences in biliary diseases. Journal of Practical Radiology. 2012; 28 (8): 1225-1227: 1234.

[17] Zhang GZ, Jiang B, Zhang D. the value of oral ferric ammonium citrate in magnetic resonance imaging of biliary and pancreatic duct. J med imaging. 2020; 21 (03): 248-250. 